\title{
Universal Fermi-Level Pinning in Transition-Metal Dichalcogenides
}

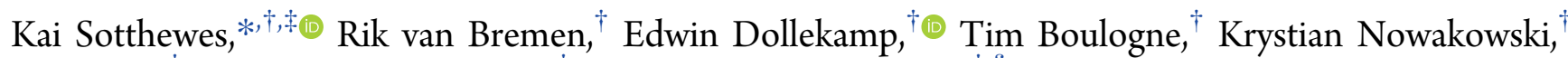
Daan Kas, ${ }^{\dagger}$ Harold J. W. Zandvliet, ${ }^{\dagger}$ and Pantelis Bampoulis $*, \dagger, \S_{\odot}$

${ }^{\dagger}$ Physics of Interfaces and Nanomaterials, MESA+ Institute for Nanotechnology, University of Twente, P.O. Box 217, 7500AE Enschede, The Netherlands

${ }^{\ddagger}$ II. Institute of Physics B and JARA-FIT, RWTH-Aachen University, 52056 Aachen, Germany

\section{Supporting Information}

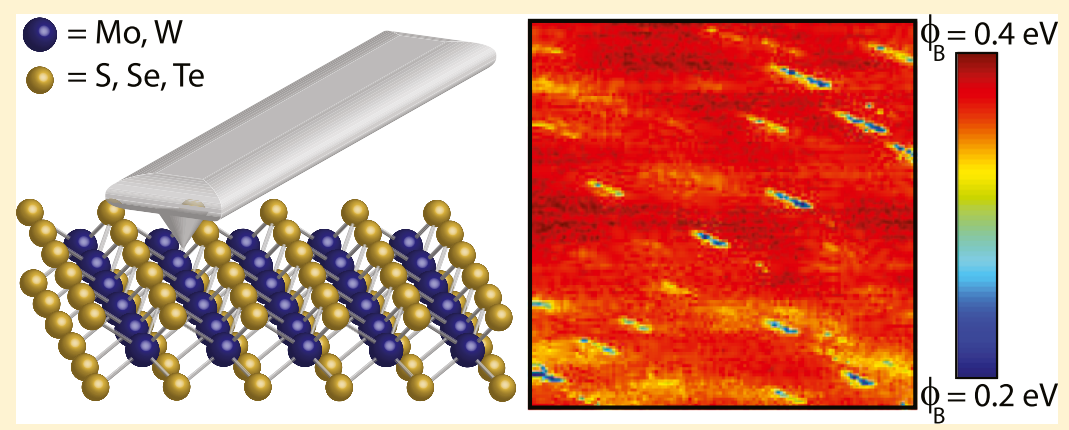

ABSTRACT: Understanding the electron transport through transition-metal dichalcogenide (TMDC)-based semiconductor/ metal junctions is vital for the realization of future TMDC-based (opto-)electronic devices. Despite the bonding in TMDCs being largely constrained within the layers, strong Fermi-level pinning (FLP) was observed in TMDC-based devices, reducing the tunability of the Schottky barrier height. We present evidence that metal-induced gap states (MIGS) are the origin for the large FLP similar to conventional semiconductors. A variety of TMDCs $\left(\mathrm{MoSe}_{2}, \mathrm{WSe}_{2}, \mathrm{WS}_{2}\right.$, and $\left.\mathrm{MoTe}_{2}\right)$ were investigated using high-spatial-resolution surface characterization techniques, permitting us to distinguish between defected and pristine regions. The Schottky barrier heights on the pristine regions can be explained by MIGS, inducing partial FLP. The FLP strength is further enhanced by disorder-induced gap states induced by transition-metal vacancies or substitutionals at the defected regions. Our findings emphasize the importance of defects on the electron transport properties in TMDC-based devices and confirm the origin of FLP in TMDC-based metal/semiconductor junctions.

\section{INTRODUCTION}

Transition-metal dichalcogenides (TMDCs) $\left(\mathrm{MX}_{2}\right.$, where $\mathrm{M}=$ Mo or $\mathrm{W}$ and $\mathrm{X}=\mathrm{S}$, Se, or Te) are two-dimensional materials with a wide range of electronic properties, for instance, insulating, semiconducting, and semi-metallic. ${ }^{1,2}$ These layered materials are constituted of hexagonal-packed transition-metal atoms sandwiched between two layers of chalcogens, with a strong intralayer covalent bond between the different atoms. These individual trilayers are held together by van der Waals bonds. The optical and electronic properties of TMDC materials depend on their composition, stacking order, and thickness. $^{2-6}$ Especially, the semiconducting TMDCs are interesting for (opto-)electronics because of their relatively high carrier mobility and their tunable band gap ${ }^{7-9}$

The metal-semiconductor junction (MSJ) is a critical component in electronic and optoelectronic devices. One of the most important parameters in the MSJs is the Schottky barrier height $\left(\mathrm{SBH}, \phi_{\mathrm{B}}\right)$, the height of the potential barrier formed at the metal-semiconductor interface, which has a significant impact on the device performance. Tuning the SBH is necessary in order to reduce the contact resistance, modulating the charge carrier type in transistors, and to enhance the selectivity of carrier extraction for photovoltaic cells. $^{10-14}$

Because of the van der Waals interlayer bonding, it was expected that the contact metal is also weakly bonded to the TMDC layer, so that the SBH would approach the SchottkyMott limit of weak pinning and could easily be tuned. ${ }^{15,16}$ However, experiments and simulations showed the opposite behavior, the SBH depends only weakly on the contact metal. ${ }^{10,17-20}$ This was attributed to Fermi-level pinning (FLP). ${ }^{17,19-21}$ The origin of the FLP has been debated for a long time. There are two well-known models describing FLP; metal-induced gap states (MIGS) ${ }^{22}$ and extrinsic disorderinduced gap states (DIGS). ${ }^{23}$ For DIGS, the FLP originates from the electronic structure of the host semiconductor within the MSJ. On the other hand, the MIGS model predicts that the metal will dominate over the substrate in pinning the Fermi level. To reveal the MIGS effect experimentally within

Received: November 12, 2018

Revised: February 13, 2019

Published: February 14, 2019 

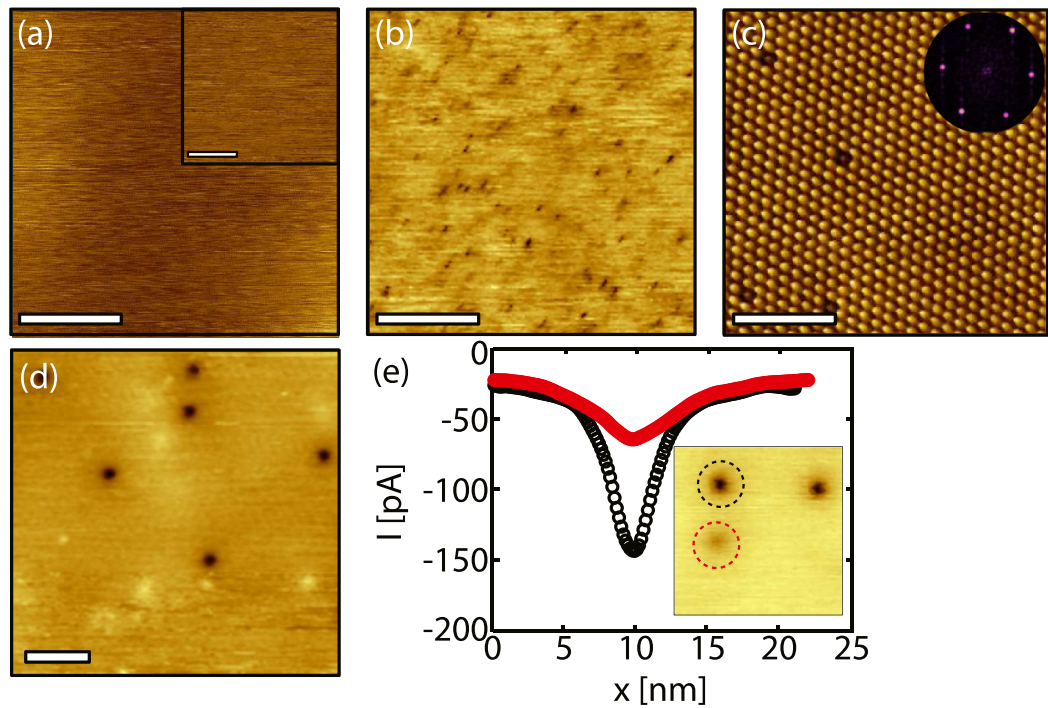

Figure 1. (a) Topographic AFM image (inset: corresponding lateral force image) of a freshly cleaved MoSe $\mathrm{e}_{2}$ crystal $\left(600 \times 600 \mathrm{~nm}^{2}\right.$, the scale bar is $200 \mathrm{~nm}$ ). (b) Simultaneously recorded C-AFM image recorded at $-1.2 \mathrm{~V}$ sample bias. (c) STM topography image of the WSe $e_{2}$ surface, revealing missing selenium atoms $\left(10 \times 10 \mathrm{~nm}^{2}\right.$, the scale bar is $\left.3 \mathrm{~nm}\right)$. The tunneling parameters are $1.1 \mathrm{nA}$ and $-0.6 \mathrm{~V}$. (d) STM topography image of dark circular features $\left(30 \times 30 \mathrm{~nm}^{2}\right.$, the scale bar is $8 \mathrm{~nm}$, tunneling parameters: $100 \mathrm{pA}$ and $\left.1.5 \mathrm{~V}\right)$. (e) Current cross section recorded with C-AFM at the dark round features induced by defects in the first and second trilayers, respectively [inset: the C-AFM image indicating the defects where the cross section was taken $\left(20 \times 20 \mathrm{~nm}^{2}\right.$, SB is $\left.1.2 \mathrm{~V}\right)$ ].

TMDCs, a reduction of the extrinsic factors, such as defects and stacking faults, is a prerequisite.

Several works have been conducted to find the origin of FLP and subsequently control its strength. ${ }^{10,17,19-21,24-26}$ Theoretical studies predicted that the FLP effect in the MSJ is mainly induced by two interface effects: first, due to modification of the metal's work function by dipole formation at the interface and second, by the introduction of gap states due to the reduction of the chalcogen/transition-metal bonding strength. $^{21}$ Later, Guo et al. performed simulations for several TMDCs, showing that the TMDC/metal junctions largely follow the MIGS model, similar to three-dimensional semiconductors despite their different structure and bonding. ${ }^{17}$ However, it is difficult to experimentally access the pinning mechanism because of the high density of surface defects, which alter the properties of these materials. ${ }^{20,24,27-29}$ To experimentally confirm the pinning mechanism of the TMDCbased MSJ, a defect-free MSJ has to be constructed.

Recently, nanoscopic MSJs have been obtained using conductive atomic force microscopy (C-AFM). In C-AFM, the tip can act as a nanoscopic metal electrode. ${ }^{6,20,30-33} I(V)$ characteristics recorded with a C-AFM of the Pt-coated tip/ $\mathrm{MoS}_{2}$ junction revealed variations in the $\mathrm{SBH} .^{31,32}$ This method was also used to investigate the effect of local defects, $^{20}$ stacking faults, ${ }^{6}$ plasma functionalization, ${ }^{34}$ and charge variations due to doping ${ }^{33,35}$ on the charge transport across the MSJ. Because of the nanoscopic size of the contact metal, small variations at the surface can be measured. Substitution or removal of the transition metal or the chalcogen atoms lead to defect sites with different electronic properties compared to the pristine regions ${ }^{28,36-38}$ including small SBH and strong FLP. ${ }^{6,20} \mathrm{On} \mathrm{MoS}_{2}$, defects located at the Mo layer enhance further the FLP strength compared to pristine surface or regions with S-vacancies. ${ }^{20}$

In this work, we use the C-AFM to construct nanoscopic MSJs on several TMDCs, in order to investigate the exact mechanism behind FLP on TMDCs. We performed high- spatial-resolution C-AFM and scanning tunneling microscopy (STM) imaging in order to characterize defects present on several TMDC surfaces $\left(\mathrm{MoSe}_{2}, \mathrm{WSe}_{2}, \mathrm{WS}_{2}\right.$, and $\left.\mathrm{MoTe}_{2}\right)$. For the studied TMDCs, we find defects with similar appearance and influence; STM reveals that chalcogen defects have almost no influence on their surroundings, whereas transition-metal defects lead to a strong localized contrast that depends on the used set point. The barrier heights of the pristine surface can be explained by the MIGS mechanism, which normally applies to three-dimensional semiconductors. Spatially resolved Schottky barrier maps reveal a decrease of the SBH and a stronger FLP at defect sites because of DIGS. The DIGS decrease the pinning factor by approximately $30-40 \%$. Our results provide new insights into the physics governing the contact properties and charge transport through the TMDCbased MSJ.

\section{RESULTS AND DISCUSSION}

Defects in TMDCs. Figure 1a shows topographic AFM and lateral force images (see the inset of Figure 1a) of a freshly cleaved $\mathrm{MoSe}_{2}$ surface. The surface appears smooth and defect-free in both images. However, within the simultaneously recorded C-AFM image, the surface is inhomogeneous when a negative voltage is applied to the sample. Multiple dark circular features (with radii ranging between 3 and $4 \mathrm{~nm}$ ) appear (see Figure $1 \mathrm{~b}$ ). The current recorded at these features is higher than that for the pristine surface. The density of the features varies between $10^{10}$ and $10^{11} \mathrm{~cm}^{-2}$ dependent on the sample and location. Imaging a region without round depressions requires a high magnification of a small area. Figure 1c shows an example of a high-resolution STM image where the atomic structure of $\mathrm{WSe}_{2}$ is observed, revealing the expected hexagonal pattern with a unit cell of $(0.33 \pm 0.02) \mathrm{nm}$. The structure is consistent with the semiconducting $2 \mathrm{H}$ phase. In addition to the observed hexagonal pattern, several missing atoms are observed. At these positions, Se atoms are missing, inducing $\mathrm{Se}$ vacancies. The electronic influence on the 


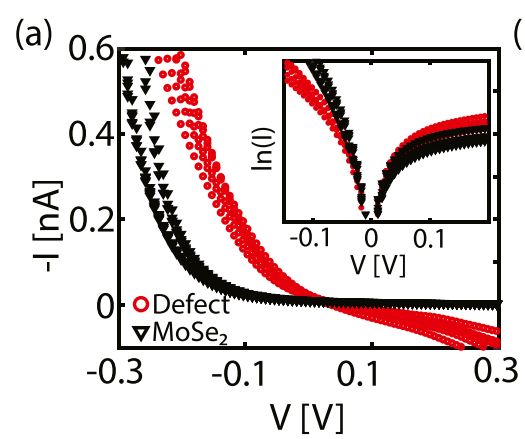

(b)
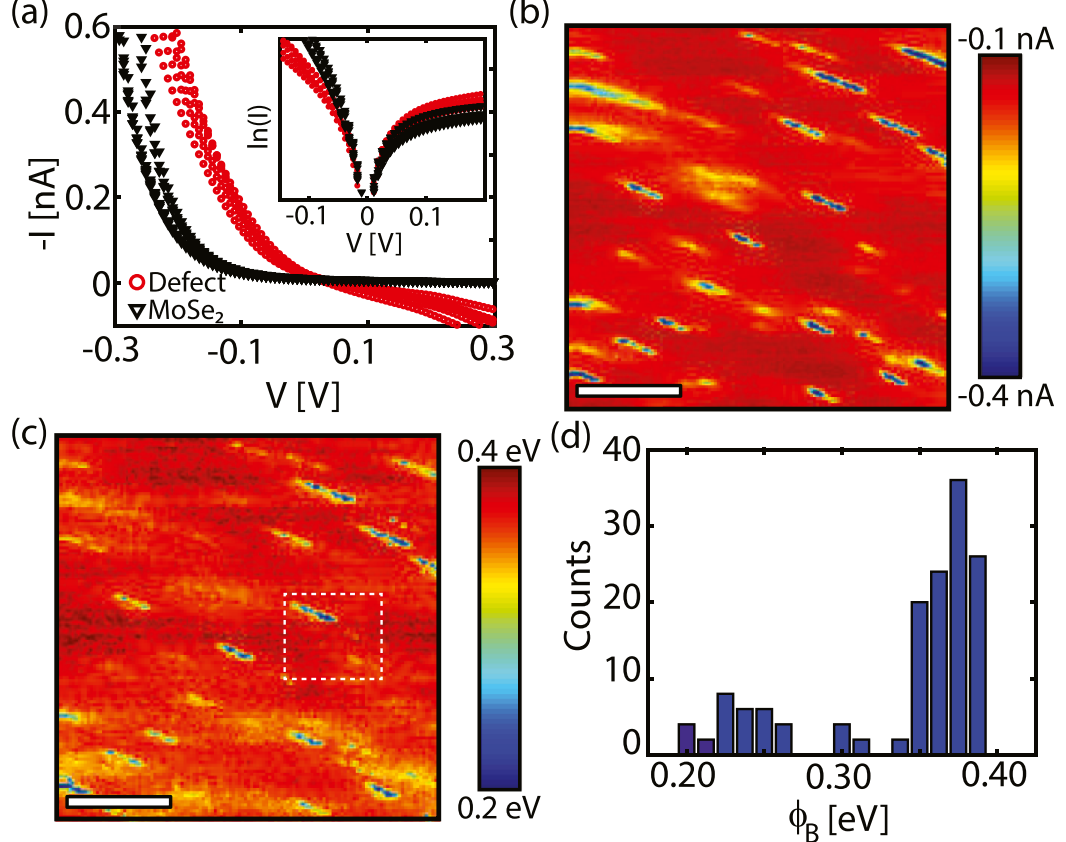

Figure 2. (a) $I(V)$ curves recorded with a Pt tip on a pristine (red) and defected (black) region of $\mathrm{MoSe}_{2}$. Inset: the corresponding semilogarithmic $I(V)$ curves from which the SB is extracted. (b) Spatially resolved current map $\left(200 \times 200 \mathrm{~nm}^{2}\right.$, scale bar $\left.50 \mathrm{~nm}\right)$ obtained via $128 \times$ 128 individual current-voltage curves, recorded from -0.6 to $1.5 \mathrm{~V}$. The map corresponds to a voltage of $-0.15 \mathrm{~V}$. (c) Extracted SBH map of the surface obtained from the individual $I(V)$ curves in (a). Significant differences in the SBH are observed at the positions of the defects in (b). For the ideality factor $(\eta)$ map, see the Supporting Information. (d) Histogram of the SBH for the white dashed box marked in (c). Three peaks are observed: $(0.37 \pm 0.03),(0.30 \pm 0.02)$, and $(0.24 \pm 0.03) \mathrm{eV}$ for the pristine region, defects located in the second trilayer and defects located in the first trilayer, respectively.

surrounding of the Se vacancies is small ( $<1 \mathrm{~nm}$ in radius), excluding them from being the origin of the dark round features in the C-AFM image (see Figure $1 \mathrm{~b}$ ). Besides, the density of the Se vacancies $\left(10^{12}\right.$ to $\left.10^{13} \mathrm{~cm}^{-2}\right)$ is 2 orders of magnitude higher compared to that of the dark circular features $\left(10^{10}\right.$ to $\left.10^{11} \mathrm{~cm}^{-2}\right)$ observed in the C-AFM.

Large-scale STM images reveal the presence of dark depressions similar to the ones observed in the C-AFM (Figure 1d). High-resolution STM images show a depression without disrupting the atomic lattice, confirming that the effect is purely electronic (see the Supporting Information). The average defect density of $10^{10}$ to $10^{11}$ per $\mathrm{cm}^{-2}$ is comparable to the density found in the C-AFM images. The appearance and density of the defects are very similar to the defects observed on $\mathrm{MoS}_{2} \cdot{ }^{20,24}$ In these studies, the origin of the dark round features was attributed to metal-like defects or impurities located underneath the outermost $S$ layer. Movacancies and antisite (Mo-substitutional) defects occur on the subsurface without a structural modification of the top $S$ layer. These defects ionize the sulfur atoms. Coulomb repulsion around these sulfur atoms leads to electron depletion and causes the appearance of the dark disk observed in both CAFM and STM. ${ }^{20,39}$ We note here that the observed defects are intrinsic in nature, as the samples have not gone through any treatment (except exfoliation) prior to scanning. In addition, similar features with similar densities were found in other types of TMDCs, such as $\mathrm{WS}_{2}{ }^{6,40}$ and $\mathrm{WSe}_{2}{ }^{29,41,42}$ attributed to impurity atoms (such as $\mathrm{Re}, \mathrm{Na}$, and $\mathrm{Li}$ ) present in the crystal. ${ }^{29,43}$ Therefore, we suggest that the dark circular features originate from metal-like defects $(\mathrm{Mo} / \mathrm{W}$ vacancies or substitutionals), which change the electronic landscape of their surroundings. Next to the dark circular defects, bright defects are observed in the STM image (Figure 1d). These defects are attributed to trapped electron states around a donor on the surface chalcogen atom layer. ${ }^{28,44}$ However, these defects are not observed in the C-AFM images.

A closer look at the metal-like defects reveals that they fall into two categories: defects with current amplitudes of approximately -150 and $-50 \mathrm{pA}$ (at a bias voltage of 1.2 V), as shown in Figure 1e. These differences are caused by the position of the metal-like defect. When located in the top $\mathrm{MoSe}_{2}$ trilayer, the electronic influence is larger compared to defects located in the second $\mathrm{MoSe}_{2}$ trilayer, as observed previously for $\mathrm{MoS}_{2}$ and conventional semiconductors. ${ }^{20,45,46}$ These results suggest that the conductance of $\mathrm{MoSe}_{2}$ can be influenced by metal-like defects in the first and second trilayers.

Similar defects are found in the C-AFM images on $\mathrm{WS}_{2}$, $\mathrm{WSe}_{2}$, and $\mathrm{MoTe}_{2}$ (see the Supporting Information). These defects have similar appearances as the metal-like defects on $\mathrm{MoS}_{2}{ }^{20}$ and $\mathrm{MoSe}_{2}$ (see Figure 1b). Earlier studies emphasized the similarities between the defect types in the different TMDCs. ${ }^{47-49}$ The energies needed to create defects within the different types of semiconducting TMDCs are comparable $^{38,50,51}$ as well as the structural conformations. ${ }^{52}$ Therefore, we conclude that the defects observed with C-AFM on the studied TMDCs are all metal-like defects.

SBHs on Pristine and Defected Regions. Because it is now possible to distinguish between defected and pristine regions (note here that the pristine regions still contains chalcogen defects), the local electron transport through nanoscopic MSJ between a Pt tip $\left(\phi_{\mathrm{M}}=5.64 \mathrm{eV}\right)$ and the semiconducting TMDC can be investigated. For zero charge transfer in the MSJ (Schottky limit), the electron SBH is given 
by the difference between the work function of the metal $\left(\phi_{\mathrm{M}}\right)$ and the electron affinity of the semiconductor $\left(\chi_{\mathrm{s}}\right)$

$$
\phi_{\mathrm{B}}=\phi_{\mathrm{M}}-\chi_{\mathrm{S}}
$$

A characteristic $I(V)$ curve for the $\mathrm{MoSe}_{2}$-based MSJ is shown in Figure 2a. The measured $I(V)$ curve on the pristine region shows a diode-like behavior, as expected for a MSJ. ${ }^{53}$ The negative sample bias is defined as the forward bias regime. For larger negative voltages, the $\mathrm{SBH}$ lowers and the current increases exponentially with the voltage. The current in the MSJ under forward bias is described by the thermionic emission model and is given by ${ }^{54}$

$$
I=I_{0} \exp \left(\frac{q V}{n k_{\mathrm{B}} T}\right)\left[1-\exp \left(\frac{-q V}{k_{\mathrm{B}} T}\right)\right]
$$

where $V$ is the applied bias, $k_{\mathrm{B}}$ is the Boltzmann constant, $T$ is the temperature, $\eta$ is the ideality factor, this is the deviation of the current transport from ideal thermal emission, and $q$ is the electron charge. For values of $V$ larger than $3 k T / q$, the second term in eq 2 becomes negligible. The reverse saturation current $\left(I_{0}\right)$ is a constant and depends on the Schottky barrier $\phi_{\mathrm{B}}$

$$
I_{0}=A A^{*} T^{2} \exp \left(-\frac{q \phi_{\mathrm{B}}}{k_{\mathrm{B}} T}\right)
$$

with $A$ being the MSJ area, that is, the tip contact area and $A^{*}$ is the Richardson constant $\left(A^{*}=\frac{4 \pi q m^{*} k_{\mathrm{B}}^{2}}{h^{3}}\right.$, with $m^{*}$ the effective mass and $h$ the Planck constant). The contact area of the Pt tip is estimated using the Hertz model ${ }^{55,56}$ and is approximately $2.7 \mathrm{~nm}^{2}$ at a load of $5-10 \mathrm{nN}$. The ideality factor is obtained from

$$
\eta=\frac{q}{k_{\mathrm{B}} T} \frac{\mathrm{d} V}{\mathrm{~d}(\ln I)}
$$

and the $\phi_{\mathrm{B}}$ for electron injection is acquired using

$$
\phi_{\mathrm{B}}=\frac{k_{\mathrm{B}} T}{q} \ln \left(\frac{A^{*} A T^{2}}{I_{0}}\right)
$$

The ideality factor is extracted from the slope of the linear part of the $\ln I(V)$ curve (like in the inset of Figure 2a). The intercept of this fit gives the saturation current which is used to extract the $\mathrm{SBH}$ according to eq 5. By applying the abovementioned equations to the recorded $I(V)$ curves in Figure 2a, the $\mathrm{SBH}$ of the pristine region is approximately $(0.37 \pm 0.02)$ $\mathrm{eV}$ and the ideality factor is 1.1 . The defected region shows a slightly higher current at the reverse bias (see Figure 2a). This is a result of a change in the SBH and ideality factor. The SBH for the defected region is $(0.24 \pm 0.04) \mathrm{eV}$ with an ideality factor of 1.5. As the $\mathrm{SBH}$ is lower, more current can pass through the junction.

The advantage of using a sharp AFM tip as the metal electrode in the MSJ is the ability to record individual $I(V)$ curves at every point of the surface. Figure $2 \mathrm{~b}$ shows a spatially resolved current map extracted from a $128 \times 128$ grid of individual $I(V)$ curves at a bias voltage of $-0.15 \mathrm{~V}$. A clear difference is found between the defects and pristine region, as already observed in the C-AFM image (see Figure $1 b^{a}$ ). We can now extract the SBH for each of the individual curves and construct a SBH map (Figure 2c). Significant inhomogeneities in the barrier height are observed. Interestingly, the $\mathrm{SBH}$ remains constant on the pristine regions of the $\mathrm{MoSe}_{2}$ surface, whereas directly above the defects, a decrease of the barrier height is measured.

The histogram in Figure $2 \mathrm{~d}$ is constructed of the current values within a specific part of the SBH map (marked with the dashed box in Figure 2c). It reveals three peaks: corresponding to the pristine region [at $(0.37 \pm 0.03) \mathrm{eV}]$, the defected region $[(0.24 \pm 0.04) \mathrm{eV}]$, and a third peak at $(0.30 \pm 0.02)$ $\mathrm{eV}$. A closer look at the current and SBH maps confirms the presence of defects located in the second trilayer, in line with the C-AFM images of Figure $1 \mathrm{~b}$. Recent calculations showed that the influence of defects on the SBH decreases as the dopant-interface distance increases. ${ }^{57}$ Therefore, defects in the second trilayer have a weaker influence on the SBH compared to defects in the first trilayer.

We have also observed defect-induced reduction of the $\mathrm{SBH}$ for $\mathrm{WS}_{2}, \mathrm{WSe}_{2}, \mathrm{MoTe}_{2}$ (see the Supporting Information), and $\mathrm{MoS}_{2}{ }^{20}$ However, the absolute values of the SBH on the pristine and defected regions differ between the different TMDCs (using the same tip). This is a result of the different electron affinities and electronegativities of the various TMDC semiconductors. According to the Schottky-Mott rule (see eq 1 ), the SBH decreases linearly with increasing electron affinity. Although a decreasing trend is found, it is not linear and the experimentally found $\mathrm{SBH}$ values do not match with the prediction of the Schottky-Mott rule.

Metal-Induced Gap States. The discrepancy between the predicted and measured SBHs has also been found in other studies for conventional as well as layered semiconductors. ${ }^{6,19,24,58-60}$ The Schottky-Mott rule assumes that there is no charge transfer between the metal and the interface states of the semiconductor. However, other studies suggested that charge transfer should also be included for the metal/twodimensional (2D) material MSJ. ${ }^{21,60}$ Generally, FLP is characterized using two different methods: (1) the Schottky-Mott approach and (2) the electronegativity concept. In the case of the Schottky-Mott approach, the $\mathrm{SBH}$ is given by

$$
\phi_{\mathrm{B}}=S\left(\phi_{\mathrm{M}}-\phi_{\mathrm{CNL}}\right)+\left(\phi_{\mathrm{CNL}}-\chi\right)
$$

where $S$ is the Schottky pinning parameter $\left(S=\mathrm{d} \phi_{\mathrm{B}} / \mathrm{d} \phi_{\mathrm{M}}\right), \chi$ is the electron affinity of the semiconductor, and $\phi_{\mathrm{CNL}}$ is the charge neutrality level with respect to the vacuum level which is given by

$$
\phi_{\mathrm{CNL}}=\frac{\chi+b}{1-S}
$$

where $b$ is the $y$-intercept of the $\phi_{\mathrm{B}}$ versus $\phi_{\mathrm{M}}$ graph. $S$ is dependent on the density of interface states per unit area $N$ and their extend into the semiconductor $\delta$ as

$$
S=\frac{1}{1+\frac{q^{2} N \delta}{\varepsilon_{\mathrm{r}} \varepsilon_{0}}}
$$

with $\varepsilon_{0} \varepsilon_{\mathrm{r}}$ being the absolute permittivity of the interface. When $S=1$, the Schottky-Mott limit is recovered (no pinning, $N \approx$ 0 , no gap states) and the $\mathrm{SBH}$ is given by eq 1 , whereas for $S=$ 0 , the $\mathrm{SBH}$ is described by the Bardeen limit of strong pinning. In the Bardeen limit, the metal Fermi level is pinned by the interface states at $\phi_{\mathrm{CNL}}$.

In the electronegativity concept, the $\mathrm{SBH}$ is determined using 
(a)

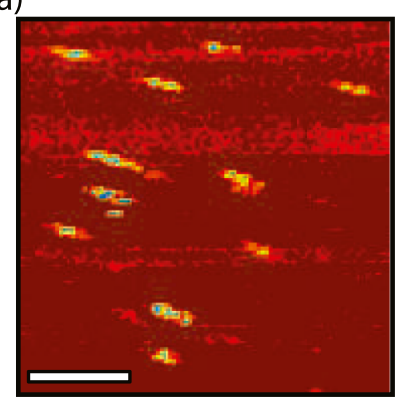

(c)

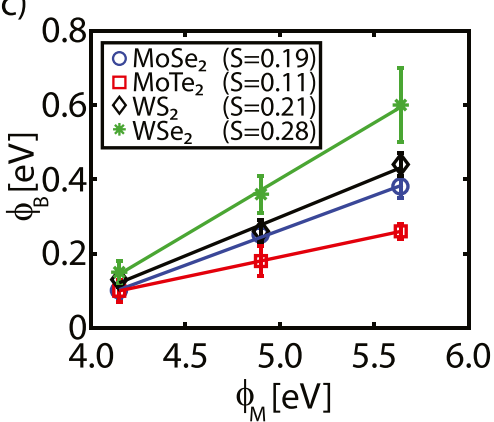

(b)
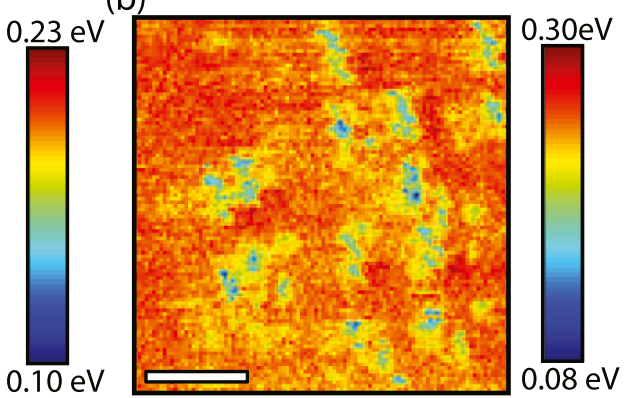

(d)

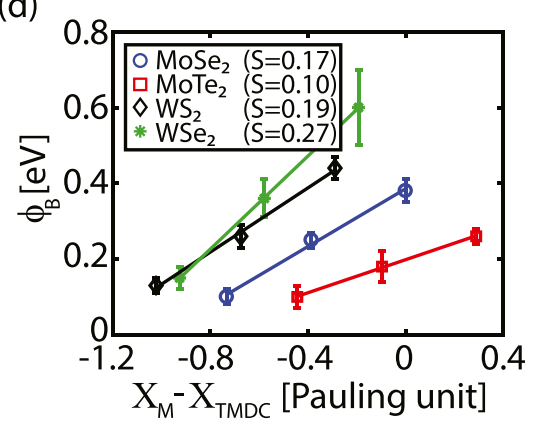

Figure 3. (a) Extracted SBH map $\left(200 \times 200 \mathrm{~nm}^{2}\right.$, the scale bar is $\left.50 \mathrm{~nm}\right)$ of the MoSe $\mathrm{C}_{2}$ surface obtained with a PtSi tip $\left(\phi_{\mathrm{M}} \approx 4.9 \mathrm{eV}\right)$. The Schottky barrier is lower on both the pristine and defected regions as compared with the measurements obtained with the Pt tip (Figure $2 \mathrm{c}$ ). (b) Extracted SBH map $\left(200 \times 200 \mathrm{~nm}^{2}\right.$, the scale bar is $\left.50 \mathrm{~nm}\right)$ for $\mathrm{MoTe}_{2}$ with a Pt tip $\left(\phi_{\mathrm{M}}=5.64\right)$. The SBH is lower as compared to MoSe 2 (Figure 2c) because of the different electron affinities $\left(\chi_{\mathrm{MoSe}_{2}}>\chi_{\mathrm{MoTe}_{2}}\right)$ and electronegativities $\left(X_{\mathrm{MoSe}_{2}}>X_{\mathrm{MoTe}_{2}}\right)$. (c,d) SBH for the pristine regions as a function of (c) metal (tip) work function $\left(\phi_{\mathrm{M}}\right)$ and $(\mathrm{d})$ electronegativity difference $\left(X_{\mathrm{M}}-X_{\mathrm{TMDC}}\right)$ for the various TMDCs. The pinning factors are extracted using eqs 6 and 9.

\section{Table 1. Pinning Factors for Both the Pristine and Defected Regions for Different TMDCs ${ }^{a}$}

$\begin{array}{lccccc}\text { TMDC } & S_{\text {pristine }} & S_{\text {pristine }}^{\prime} & S_{\text {pristine }}^{\prime} \rightarrow S_{\text {pristine }} & S_{\text {defect }} & S_{\text {defect }}^{\prime} \\ \mathrm{MoS}_{2}^{20} & 0.30 \pm 0.03 & 0.65 \pm 0.07 & 0.28 \pm 0.03 & 0.11 \pm 0.02 & 0.22 \pm 0.04 \\ \mathrm{MoSe}_{2} & 0.19 \pm 0.03 & 0.38 \pm 0.04 & 0.17 \pm 0.02 & 0.11 \pm 0.02 & 0.23 \pm 0.04 \\ \mathrm{MoTe}_{2} & 0.11 \pm 0.02 & 0.21 \pm 0.03 & 0.10 \pm 0.02 & 0.04 \pm 0.01 & 0.10 \pm 0.02 \\ \mathrm{WS}_{2} & 0.21 \pm 0.03 & 0.42 \pm 0.06 & 0.19 \pm 0.03 & 0.08 \pm 0.01 & 0.08 \pm 0.01 \\ \mathrm{WSe}_{2} & 0.28 \pm 0.03 & 0.61 \pm 0.07 & 0.27 \pm 0.03 & 0.09 \pm 0.02 & 0.04 \pm 0.01\end{array}$

${ }^{a}$ The pinning factors are obtained via two different concepts: (1) Schottky-Mott relation $(S)$ and $(2)$ electronegativity concept $\left(S^{\prime}\right)$. $S^{\prime}$ is converted to $S\left(S^{\prime} \rightarrow S\right)$ using the relation $S^{\prime}=2.27 S .^{62}$ The pinning factors are extracted from Figures $3 c, d$ and $5 \mathrm{a}, \mathrm{b}$.

$$
\phi_{\mathrm{B}}=\phi_{\mathrm{CNL}}^{\prime}+S^{\prime}\left(X_{\mathrm{M}}-X_{\mathrm{TMDC}}\right)
$$

with $S^{\prime}$ the experimentally obtained pinning factor $\left(S^{\prime}=\mathrm{d} \phi_{\mathrm{B}} /\right.$ $\left.\mathrm{d} X^{61,62}\right), X_{\mathrm{M}}$ the electronegativity of the metal, $X_{\mathrm{TMDC}}$ the electronegativity of the TMDC, and $\phi_{\mathrm{CNL}}^{\prime}$ the charge neutrality level with respect to the vacuum level. The difference between the concepts is the use of a surface property (work function and electron affinity) for the Schottky-Mott concept and a bulk property (electronegativity) for the electronegativity concept.

In order to determine the pinning factors and charge neutrality levels of the different TMDCs, the dependence of the $\phi_{\mathrm{B}}$ on the tip work function and electronegativity should be systematically studied. Therefore, we have performed the same measurements with two additional AFM tips: a PtSi tip $\left(\phi_{\mathrm{M}} \approx\right.$ $4.9 \mathrm{eV}, X_{\mathrm{M}} \approx 2.08$, contact area $5.7 \mathrm{~nm}^{2}$ ) and a highly n-doped Si tip $\left(\phi_{\mathrm{M}} \approx 4.15 \mathrm{eV}, X_{\mathrm{M}} \approx 1.9\right.$, contact area $\left.1.5 \mathrm{~nm}^{2}\right)$. For both tips, similar maps are obtained as for the Pt tip. Figure 3a shows the extracted $\mathrm{SBH}$ map for a PtSi tip on $\mathrm{MoSe}_{2}$. A decrease of approximately $33 \%$ in the $\mathrm{SBH}$ is observed compared to the results obtained with the Pt tip on $\mathrm{MoSe}_{2}$ for both the defects $[(0.14 \pm 0.03) \mathrm{eV}]$ and the pristine region
$[(0.24 \pm 0.02) \mathrm{eV}]$. This is attributed to the lower work function and electronegativity of the $\mathrm{PtSi}$ tip. In addition, defects in the second trilayer are no longer observed. The absolute value of the SBH decreases (as well as the absolute difference) making it no longer possible to resolve the presence of defects in the second trilayer.

Measurements performed with a Pt tip on $\mathrm{MoTe}_{2}$ (Figure $3 b)$ reveal a lower $\mathrm{SBH}$ as compared to the same measurements on $\mathrm{MoSe}_{2}$ (Figure 2c). The distinct difference between the $\mathrm{SBHs}$ is in line with the different electron affinities and electronegativities of $\mathrm{MoSe}_{2}$ and $\mathrm{MoTe}_{2}$, where $\chi_{\mathrm{MoSe}_{2}}>$ $\chi_{\mathrm{MoTe}_{2}}{ }^{63,64}$ and $X_{\mathrm{MoSe}_{2}}>X_{\mathrm{MoTe}_{2}}$. According to the SchottkyMott rule (eq 1), the $\mathrm{MoTe}_{2}$ should have a lower SBH. However, the absolute change in the SBHs cannot be explained using the Schottky-Mott rule, as charge transfer is not included in the model.

To explain the discrepancy between the obtained SBH values and the Schottky-Mott rule, the pinning factors and charge neutrality levels of the studied TMDCs have to be obtained. Figure $3 \mathrm{c}$ plots the experimentally obtained $\mathrm{SBH}$ for the pristine region of the studied TMDCs versus the metal 
work function of the used tips. The SBHs for all of the TMDCs depend linearly on the work function of the metal, despite the different interactions between the TMDCs and the various tips. ${ }^{59}$ In all four cases (see Table 1), a strong FLP is observed, implying a strong interaction between the metal contact and the TMDC. ${ }^{17,21,80}$ The observed pinning factor of the pristine surface of $\mathrm{WSe}_{2}(S=0.28)$ and $\mathrm{MoTe}_{2}(S=0.11)$ is in good agreement with the theoretically predicted pinning factors. ${ }^{17}$

The SBHs for $\mathrm{MoSe}_{2}(S=0.19)$ and $\mathrm{WS}_{2}(S=0.21)$ are slightly lower as compared to theory but still agree reasonably well. ${ }^{17}$ The small deviation may be a result of the doping level. In our study, all of the TMDCs are n-type doped (see the Supporting Information), whereas for many calculations, ptype-doped TMDCs are used. ${ }^{17}$ The different pinning factor values emphasize that a different degree of pinning occurs for different TMDCs, which from eq 8 implies that a different density of gap states is causing the FLP in each case (the penetration depth $\delta$ is approximately constant for all of the TMDCs). ${ }^{17,59,60}$

The linear dependence of the SBH as a function of the metal work function (Figure 3c) shows that an electric-dipole layer exists at the TMDC-Schottky contacts. ${ }^{21,60}$ Together with the strong FLP between the metal and the TMDC, it is suggested that the band-structure line up at the Schottky contact is determined by the same mechanism as for conventional semiconductors, which is described by the MIGS model. Within the MIGS model, the main origin of the gap states, which causes the pinning, is the evanescent states of the metal plane waves as they decay in the semiconductor band gap. Later, Gong et al. ${ }^{21}$ refined the exact pinning mechanism for TMDCs. They claimed that two interface effects induce FLP: first, the dipole formation is altering the work function and second, interface interactions weaken the transition-metal/ chalcogen bonding, which introduces the formation of gap states.

To explore the effect of dipole formation in more detail, the $\mathrm{SBH}$ is plotted as a function of the respective electronegativity $(X)$ difference between the metal and the semiconductor (see Figure 3d). A linear trend is observed in agreement with Figure 3c. The extracted pinning factors $S^{\prime}$ are consistent with the pinning factors in Table 1 . The obtained charge neutrality levels for all four TMDCs are located in proximity of the conduction band (see Table 2), which is expected for n-type Schottky barriers. The charge neutrality levels for the electronegativity concept do not match with the charge neutrality levels obtained using eq 7 , but are comparable to previously found charge neutrality levels for other layered semiconductor compounds. ${ }^{65}$ The difference between the two

Table 2. Charge Neutrality Levels (with Respect to Conduction Band Minimum) for Both the Pristine and Defected Regions for Different TMDCs ${ }^{a}$

$\begin{array}{lcccc}\mathrm{TMDC} & \begin{array}{c}\phi_{\mathrm{CNL}, \text { pristine }}[\mathrm{eV}] \\ \mathrm{MoS}_{2}{ }^{20}\end{array} & \begin{array}{c}\phi^{\prime}{ }_{\text {CNL,pristine }} \\ {[\mathrm{eV}]}\end{array} & \begin{array}{c}\phi_{\text {CNL,defect }} \\ {[\mathrm{eV}]}\end{array} & \begin{array}{c}\phi^{\prime}{ }_{\text {CNL,defect }} \\ {[\mathrm{eV}]}\end{array} \\ \mathrm{MoSe}_{2} & 0.07 \pm 0.02 & 0.6 \pm 0.05 & 0.13 \pm 0.03 & 0.22 \pm 0.03 \\ \mathrm{MoTe}_{2} & 0.06 \pm 0.02 & 0.39 \pm 0.05 & 0.13 \pm 0.2 & 0.25 \pm 0.04 \\ \mathrm{WS}_{2} & 0.11 \pm 0.02 & 0.55 \pm 0.05 & 0.07 \pm 0.1 & 0.24 \pm 0.03 \\ \mathrm{WSe}_{2} & 0.03 \pm 0.01 & 0.71 \pm 0.09 & 0.08 \pm 0.2 & 0.28 \pm 0.04\end{array}$

${ }^{a_{\text {The }}}$ charge neutrality levels are obtained via two different concepts: (1) Schottky-Mott relation $\left(\phi_{\mathrm{CNL}}\right)$ and (2) electronegativity concept $\left(\phi_{\mathrm{CNL}}^{\prime}\right) . \phi_{\mathrm{CNL}}$ and $\phi_{\mathrm{CNL}}^{\prime}$ are determined using the relations 7 and 9. concepts is attributed to the use of surface (work function and electron affinity) and bulk properties (electronegativities). The preference for using the electronegativity rather than the work function for calculating the charge neutrality level was led by the difference between the internal work function related to the electronegativity and the measured work function due to the additional electronic contribution from the surface dipole of the metal. ${ }^{60,66,67}$

When the electronegativities of both the metal and the semiconductor are equal, no charge transfer is expected to occur at the interface. The linear dependence observed between the $\mathrm{SBH}$ and the electronegativity difference demonstrates that dipoles indeed exist within metal/TMDC interfaces and follow the same trend as conventional semiconductors. ${ }^{60}$ In most cases, the semiconductor is more electronegative than the metal resulting in an electron accumulation on the TMDC side and depletion on the metal side of the interface. In other words, charge transfer from the metal toward the more electronegative TMDC occurs, increasing the work function of the metal in agreement with refs 21 and 68. Only for the case of a metal tip on $\mathrm{MoTe}_{2}$, the dipole moment switches, reducing the work function of the metal.

Further evidence that TMDC-based MSJs follow the MIGS model is presented in Figure 4, where the experimentally

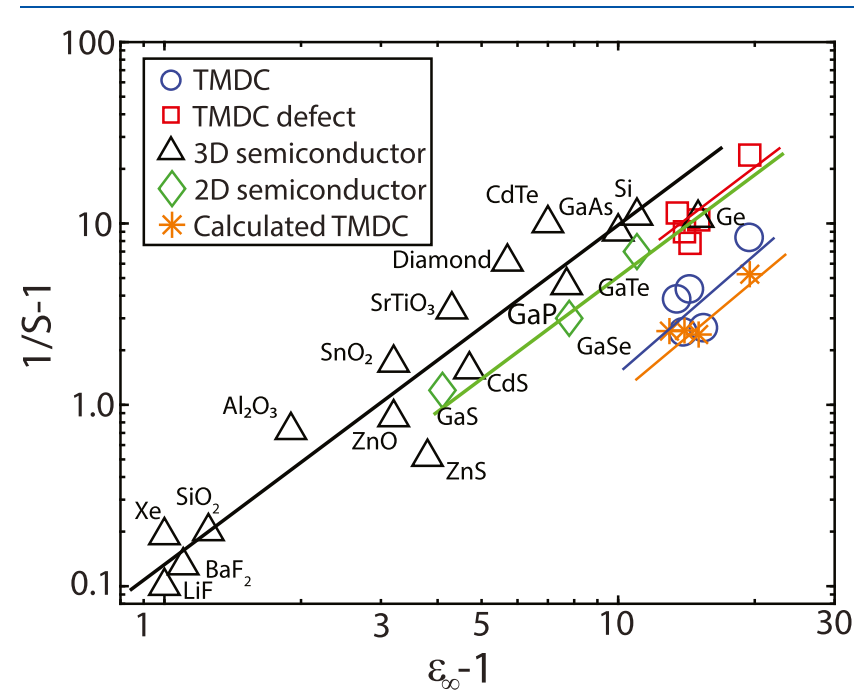

Figure 4. Experimental values of the pinning factor $(S)$ for conventional semiconductors ${ }^{71}(\triangle)$, for the $2 \mathrm{D}$ conventional semiconductor $^{72}(\diamond)$, for TMDCs in this study for the pristine region $(\bigcirc)$ and defected region $(\square)$ and calculated $\operatorname{TMDCs}^{17}(*)$ as a function of the optical dielectric constant $\left(\varepsilon_{\infty}\right)$. The solid lines are a guide to the eye based on eq 10 . For all the data point labels, see the Supporting Information.

obtained inverse pinning factor is plotted versus the optical part of the dielectric constant, $\varepsilon_{\infty}$. In 1978, Schlüter proposed that there is a relation between the $\mathrm{SBH}$ of conventional semiconductors and their dielectric constant. ${ }^{66}$ Later, Tersoff ${ }^{69}$ suggested a correlation with the optical dielectric constant, $\varepsilon_{\infty}$, which was later extended by Mönch, ${ }^{70}$ who found that $S$ obeys the empirical relation

$$
S \propto \frac{1}{1+0.1\left(\varepsilon_{\infty}-1\right)^{2}}
$$


The solid lines in Figure 4 clearly show that all types of semiconductors (including TMDCs) follow the relation given in eq 10 and that FLP in TMDCs can be described by the MIGS model. ${ }^{17}$ The vertical shift of the TMDC data $(\square$ in Figure 4) with respect to the conventional semiconductors $(\triangle$ in Figure 4) to lower pinning factors is caused by a lower net density of gap states $(N)$ in eq 8 . Using eq 8 , the density of gap states can be estimated. Assuming that the penetration depth is $\delta \approx 3 \AA^{59}$ and $\varepsilon \approx 7^{73}$ (note here: this is not $\varepsilon_{\infty}$ ) for the various TMDCs (except $\mathrm{MoTe}_{2}$ ), the density of gap states $N$ is estimated at $1 \times 10^{14}$ states $/ \mathrm{eV} \mathrm{cm} \mathrm{cm}^{2}$. The obtained density is lower than the theoretically calculated value $2.3 \times 10^{14}$ states/ $\mathrm{eV} \mathrm{cm}^{2}$ for a jellium/diamond interface ${ }^{67,74}$ (in good agreement with Figure 4, where $S_{\text {diamond }}<S_{\text {TMDC }}$ ). From Figure 4, the low pinning factor for $\mathrm{MoTe}_{2}$ with respect to the other TMDCs is also explained. The number of MIGS is inversely dependent on the width of the optical band gap. ${ }^{61}$ As $\mathrm{MoTe}_{2}$ has a smaller optical band gap than the other TMDCs, more MIGS are present in the Schottky junction and a stronger FLP is observed.

Furthermore, it was suggested that the presence of defects gives rise to extra pinning because of DIGS. ${ }^{10,17,24,75}$ Figure $5 \mathrm{a}, \mathrm{b}$ shows the $\mathrm{SBH}$ for the defected regions of the TMDCs as a function of the metal work function and the electronegativity difference, respectively. Tables 1 and 2 give an overview of the extracted pinning factor and charge neutrality levels. In the investigated cases, the FLP is stronger at the positions of the defects. In the majority of the cases, a drop of approximately $30-40 \%$ in the pinning factor is observed for defected regions with respect to the pristine surface. This is the result of DIGS. Defects lead to the formation of dangling bonds, which result in variations of the transition-metal orbital symmetry. ${ }^{26}$ Consequently, some empty states become occupied and move into the band gap to form DIGS. ${ }^{76,77}$ The effect of the defects on the $\mathrm{SBH}$, pinning factor, and charge neutrality level is independent of the TMDC type (complimentary with the observation in the current image of the C-AFM and in the STM), implying that the defects are of the same origin. As already mentioned earlier, these defects are most probably transition-metal vacancies or substitutionals. ${ }^{29,36,37}$

Several experimental ${ }^{10,24}$ and theoretical studies ${ }^{17,26,38}$ also observed an increase in FLP because of defects. In these studies, chalcogen vacancies were assigned as the origin of the DIGS. Similar to $\mathrm{MoS}_{2},{ }^{20}$ the strong FLP in this study is not caused by chalcogen vacancies as these are (1) still present on the pristine surface, (2) do not influence the conductivity, and (3) do not significantly alter their surroundings within the CAFM and STM images (see Figure 1c). In addition, the pinning factors of the pristine regions (including chalcogen defects) are in good agreement with theory. In contrast, the pinning factor of the defected regions (induced by transitionmetal vacancies or substitutionals) corresponds well to the pinning factors found in previously reported experimental studies. $^{19,20}$

When the pinning factors of the defected regions for the various TMDCs are plotted as a function of the optical dielectric constant ( $\square$ in Figure 4), the same trend is observed for the pristine regions. The points are shifted to lower pinning factors as the DIGS increase the net density of gap states $(N)$. The defected TMDC curve coincides with the curve for conventional semiconductors, implying that the net density of gap states $(N)$ is similar.
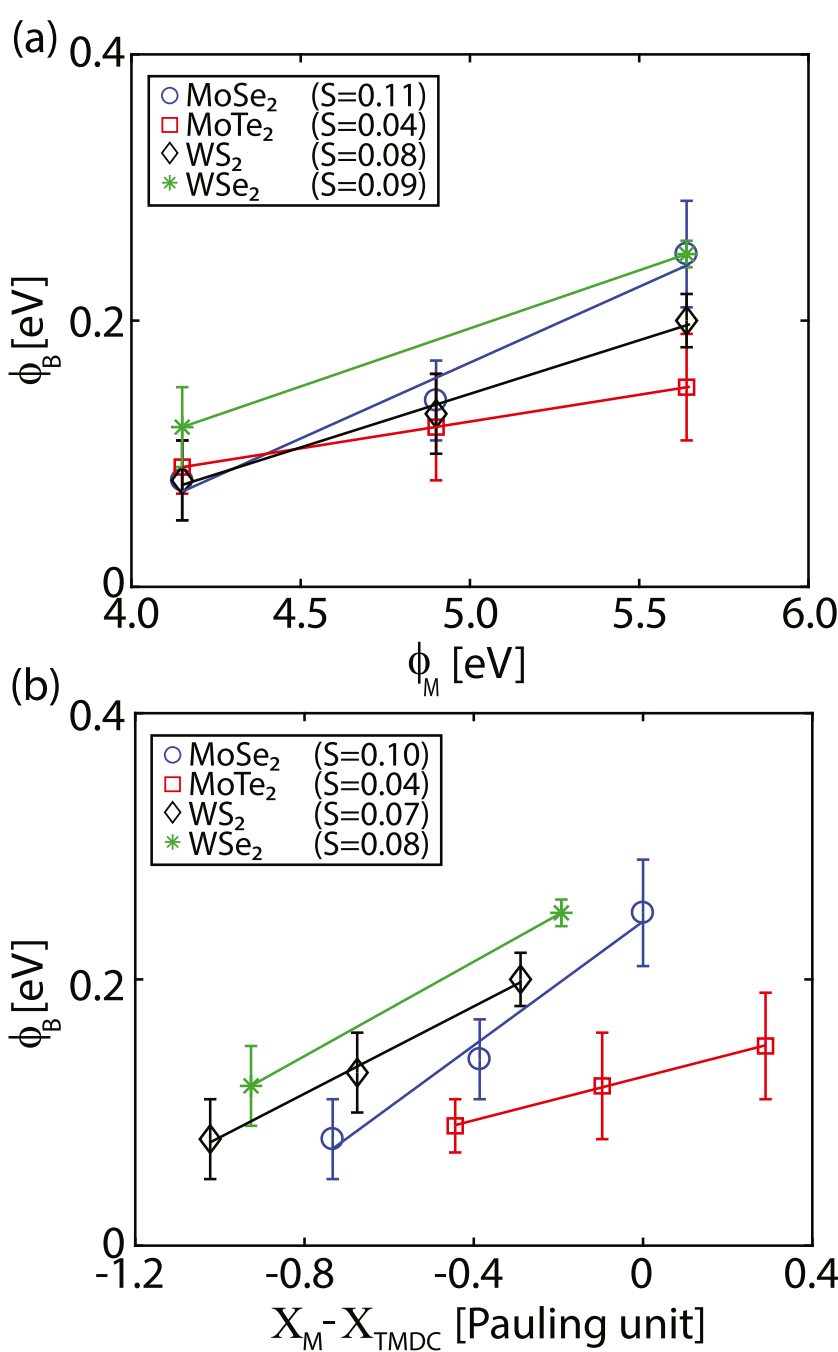

Figure 5. (a,b) SBH for the defected regions as a function of (a) metal (tip) work function $\left(\phi_{\mathrm{M}}\right)$ and (b) electronegativity difference $\left(X_{\mathrm{M}}-X_{\mathrm{TMDC}}\right)$ for the various TMDCs. The pinning factors are extracted using eqs 6 and 9 .

Unfortunately, FLP still occurs when MSJs are constructed using a conventional metal and TMDCs as a semiconductor, on contrary to the expectations based on their weak bonding with other materials. Several studies proposed promising paths to reduce FLP in TMDCs, such as the use of buffer layers ${ }^{25}$ or the use of other van der Waals materials as the contact material. ${ }^{78,79}$ Even in these junctions, subsurface defects will lower the SBH and will have a tremendous impact on the device performance. Furthermore, the effect of defects will be more pronounced when electronics are scaled down, wherein the contact area is just a few nanometers. Therefore, the conductance variations induced by transition-metal vacancies (or substitutionals) should be considered in device characterization and predictability.

\section{CONCLUSIONS}

In summary, using C-AFM and STM, we have studied the underlying mechanisms behind FLP in various TMDCs. Using a nanoscopic MSJ, we were able to distinguish between intrinsic and extrinsic factors responsible for the FLP. Spatially resolved $\mathrm{SBH}$ maps reveal a substantial difference between pristine and defected regions of the TMDC surface depending 
on the work function of the metal AFM tip. A strong FLP was observed on the pristine regions which can be explained by the MIGS model in line with conventional semiconductors. The extracted pinning factors are in good agreement with the theoretically predicted values. ${ }^{17}$ The defects induce an even stronger FLP (30-40\% increase) because of DIGS. The pinning factor found in defected regions agrees well with the experimental values obtained in previous studies where large metal contacts were used. ${ }^{19}$ Our findings show that on van der Waals semiconductor surfaces without dangling bonds, the Fermi level is still heavily pinned because of MIGS. Moreover, the FLP is enhanced in the surroundings of subsurface defects because of DIGS. These defects can provide an alternative route for surface functionalization for device applications.

\section{METHODS}

The TMDCs were mechanically cleaved and subsequently inserted in the AFM environmental chamber. The measurements were performed in $\mathrm{N}_{2}$ environment by continuously purging with $\mathrm{N}_{2}$ gas in order to reduce the influence from contaminants. The samples were imaged in the contact mode with an Agilent 5100 AFM using conductive probes. For the CAFM measurements, the tip was grounded, whereas a bias voltage was applied to the various TMDC crystals. The conductive tips used are: Pt tips (12Pt400B, Rocky Mountain Nanotechnology), PtSi tips (PtSi-CONT, NANOSENSORS), and highly n-doped Si tips (Hi-res-C14/Cr-Au. MikroMasch, resistivity: $0.01-0.025 \Omega \mathrm{cm}$ ) (for detailed information see the Supporting Information). STM and scanning tunneling spectroscopy measurements were performed in an Omicron low-temperature STM operating at a base pressure below $1 \times$ $10^{-10} \mathrm{mbar}$ and a base temperature of $77 \mathrm{~K}$.

\section{ASSOCIATED CONTENT}

\section{S Supporting Information}

The Supporting Information is available free of charge on the ACS Publications website at DOI: 10.1021/acs.jpcc.8b10971.

Experimental configuration, cantilever and material properties, defect types, ideality factor, $\mathrm{MoSe}_{2}, \mathrm{MoTe}_{2}$, $\mathrm{WS}_{2}, \mathrm{WSe}_{2}$, and pinning factor versus optical dielectric constant $(\mathrm{PDF})$

\section{AUTHOR INFORMATION}

\section{Corresponding Authors}

*E-mail: k.sotthewes@utwente.nl (K.S.).

*E-mail: bampoulis@ph2.uni-koeln.de (P.B.).

ORCID

Kai Sotthewes: 0000-0003-2073-6958

Edwin Dollekamp: 0000-0001-8956-5785

Pantelis Bampoulis: 0000-0002-2347-5223

\section{Present Address}

${ }^{\S}$ II. Physikalisches Institut, Universität zu Köln, Zülpicher Straße 77, 50937 Köln, Germany.

\section{Notes}

The authors declare no competing financial interest.

\section{ACKNOWLEDGMENTS}

This work is part of the research programme on 2D semiconductor crystals with project number FV157-TWOD, which is financed by the Netherlands Organization for Scientific Research (NWO). E.D. thanks the Netherlands
Center for Multiscale Catalytic Energy Conversion, MCEC, an NWO Gravitation programme funded by the Ministry of Education, Culture and Science of the government of the Netherlands.

\section{ADDITIONAL NOTE}

${ }^{a_{T}}$ The defects are displayed elongated because of drift in the AFM.

\section{REFERENCES}

(1) Wang, Q. H.; Kalantar-Zadeh, K.; Kis, A.; Coleman, J. N.; Strano, M. S. Electronics and Optoelectronics of Two-Dimensional Transition Metal Dichalcogenides. Nat. Nanotechnol. 2012, 7, 699.

(2) Duan, X.; Wang, C.; Pan, A.; Yu, R.; Duan, X. Two-Dimensional Transition Metal Dichalcogenides as Atomically Thin Semiconductors: Opportunities and Challenges. Chem. Soc. Rev. 2015, 44, 88598876.

(3) Splendiani, A.; Sun, L.; Zhang, Y.; Li, T.; Kim, J.; Chim, C.-Y.; Galli, G.; Wang, F. Emerging Photoluminescence in Monolayer $\mathrm{MoS}_{2}$. Nano Lett. 2010, 10, 1271-1275.

(4) He, J.; Hummer, K.; Franchini, C. Stacking effects on the electronic and optical properties of bilayer transition metal dichalcogenides $\mathrm{MoS}_{2}, \mathrm{MoSe}_{2}, \mathrm{WS}_{2}$, and $\mathrm{WSe}_{2}$. Phys. Rev. B: Condens. Matter Mater. Phys. 2014, 89, 075409.

(5) Kutana, A.; Penev, E. S.; Yakobson, B. I. Engineering Electronic Properties of Layered Transition-Metal Dichalcogenide Compounds Through Alloying. Nanoscale 2014, 6, 5820-5825.

(6) Bampoulis, P.; Sotthewes, K.; Siekman, M. H.; Zandvliet, H. J. W. Local Conduction in $\mathrm{Mo}_{x} \mathrm{~W}_{1-x} \mathrm{Se}_{2}$ : The Role of Stacking Faults, Defects, and Alloying. ACS Appl. Mater. Interfaces 2018, 10, 1321813225 .

(7) Radisavljevic, B.; Radenovic, A.; Brivio, J.; Giacometti, V.; Kis, A. Single-layer MoS2 transistors. Nat. Nanotechnol. 2011, 6, 147.

(8) Kim, S.; Konar, A.; Hwang, W.-S.; Lee, J. H.; Lee, J.; Yang, J.; Jung, C.; Kim, H.; Yoo, J.-B.; Choi, J.-Y.; et al. High-Mobility and Low-Power Thin-Film Transistors Based on Multilayer $\mathrm{MoS}_{2}$ crystals. Nat. Commun. 2012, 3, 1011.

(9) Choi, W.; Choudhary, N.; Han, G. H.; Park, J.; Akinwande, D.; Lee, Y. H. Recent Development of Two-Dimensional Transition Metal Dichalcogenides and Their Applications. Mater. Today 2017, 20, 116-130.

(10) Das, S.; Chen, H.-Y.; Penumatcha, A. V.; Appenzeller, J. High Performance Multi-layer $\mathrm{MoS}_{2}$ Transistors with Scandium Contacts. Nano Lett. 2012, 13, 100.

(11) Pradhan, N. R.; Rhodes, D.; Zhang, Q.; Talapatra, S.; Terrones, M.; Ajayan, P. M.; Balicas, L. Intrinsic Carrier Mobility of MultiLayered $\mathrm{MoS}_{2}$ Field-Effect Transistors on $\mathrm{SiO}_{2}$. Appl. Phys. Lett. 2013, $102,123105$.

(12) Bernardi, M.; Palummo, M.; Grossman, J. C. Extraordinary Sunlight Absorption and One Nanometer Thick Photovoltaics Using Two-Dimensional Monolayer Materials. Nano Lett. 2013, 13, 36643670.

(13) Allain, A.; Kang, J.; Banerjee, K.; Kis, A. Electrical Contacts to Two-Dimensional Semiconductors. Nat. Mater. 2015, 14, 1195.

(14) Schulman, D. S.; Arnold, A. J.; Das, S. Contact Engineering for 2D Materials and Devices. Chem. Soc. Rev. 2018, 47, 3037-3058.

(15) Lince, J. R.; Carré, D. J.; Fleischauer, P. D. Schottky-Barrier Formation on a Covalent Semiconductor without Fermi-Level Pinning: The Metal- $\mathrm{MoS}_{2}(0001)$ Interface. Phys. Rev. B: Condens. Matter Mater. Phys. 1987, 36, 1647-1656.

(16) Jaegermann, W.; Ohuchi, F. S.; Parkinson, B. A. Interaction of $\mathrm{Cu}, \mathrm{Ag}$ and $\mathrm{Au}$ with van der Waals faces of WS, and SnS2. Surf. Sci. 1988, 201, 211-227.

(17) Guo, Y.; Liu, D.; Robertson, J. 3D Behavior of Schottky Barriers of 2D Transition-Metal Dichalcogenides. ACS Appl. Mater. Interfaces 2015, 7, 25709.

(18) Yuan, H.; Cheng, G.; You, L.; Li, H.; Zhu, H.; Li, W.; Kopanski, J. J.; Obeng, Y. S.; Walker, A. R. H.; Gundlach, D. J.; et al. Influence of 
Metal-MoS2 Interface on MoS2 Transistor Performance: Comparison of $\mathrm{Ag}$ and Ti Contacts. ACS Appl. Mater. Interfaces 2015, 7, 11801187.

(19) Kim, C.; Moon, I.; Lee, D.; Choi, M. S.; Ahmed, F.; Nam, S.; Cho, Y.; Shin, H.-J.; Park, S.; Yoo, W. J. Fermi Level Pinning at Electrical Metal Contacts of Monolayer Molybdenum Dichalcogenides. ACS Nano 2017, 11, 1588-1596.

(20) Bampoulis, P.; van Bremen, R.; Yao, Q.; Poelsema, B.; Zandvliet, H. J. W.; Sotthewes, K. Defect Dominated Charge Transport and Fermi Level Pinning in $\mathrm{MoS}_{2} /$ Metal Contacts. ACS Appl. Mater. Interfaces 2017, 9, 19278-19286.

(21) Gong, C.; Colombo, L.; Wallace, R. M.; Cho, K. The Unusual Mechanism of Partial Fermi Level Pinning at Metal - $\mathrm{MoS}_{2}$ Interfaces. Nano Lett. 2014, 14, 1714.

(22) Heine, V. Theory of Surface States. Phys. Rev. 1965, 138, A1689.

(23) Hasegawa, H.; Ohno, H. Unified Disorder Induced Gap State Model for Insulator-Semiconductor and Metal-Semiconductor Interfaces. J. Vac. Sci. Technol., B: Microelectron. Process. Phenom. 1986, 4, $1130-1138$

(24) McDonnell, S.; Addou, R.; Buie, C.; Wallace, R. M.; Hinkle, C. L. Defect-Dominated Doping and Contact Resistance in $\mathrm{MoS}_{2}$. ACS Nano 2014, 8, 2880.

(25) Kim, G.-S.; Kim, S.-H.; Park, J.; Han, K. H.; Kim, J.; Yu, H.-Y. Schottky Barrier Height Engineering for Electrical Contacts of Multilayered $\mathrm{MoS}_{2}$ Transistors with Reduction of Metal-Induced Gap States. ACS Nano 2018, 12, 6292-6300.

(26) Su, J.; Feng, L.; Zhang, Y.; Liu, Z. Defect Induced Gap States in Monolayer $\mathrm{MoS}_{2}$ Control the Schottky Barriers of Pt-mMoS Interfaces. Appl. Phys. Lett. 2017, 110, 161604.

(27) Hong, J.; Hu, Z.; Probert, M.; Li, K.; Lv, D.; Yang, X.; Gu, L.; Mao, N.; Feng, Q.; Xie, L.; et al. Exploring Atomic Defects in Molybdenum Disulphide Monolayers. Nat. Commun. 2015, 6, 6293.

(28) Addou, R.; Colombo, L.; Wallace, R. M. Surface Defects on Natural $\mathrm{MoS}_{2}$. ACS Appl. Mater. Interfaces 2015, 7, 11921-11929.

(29) Addou, R.; Wallace, R. M. Surface Analysis of WSe $\mathrm{W}_{2}$ Crystals: Spatial and Electronic Variability. ACS Appl. Mater. Interfaces 2016, 8, 26400-26406.

(30) Fu, D.; Zhou, J.; Tongay, S.; Liu, K.; Fan, W.; King Liu, T.-J.; $\mathrm{Wu}, \mathrm{J}$. Mechanically Modulated Tunneling Resistance in Monolayer $\mathrm{MoS}_{2}$. Appl. Phys. Lett. 2013, 103, 183105.

(31) Giannazzo, F.; Fisichella, G.; Piazza, A.; Agnello, S.; Roccaforte, F. Nanoscale Inhomogeneity of the Schottky Barrier and Resistivity in $\mathrm{MoS}_{2}$ Multilayers. Phys. Rev. B: Condens. Matter Mater. Phys. 2015, 92, 081307.

(32) Giannazzo, F.; Fisichella, G.; Piazza, A.; Di Franco, S.; Oliveri, I. P.; Agnello, S.; Roccaforte, F. Current injection from metal to $\mathrm{MoS}_{2}$ Probed at Nanoscale by Conductive Atomic Force Microscopy. Mater. Sci. Semicond. Process. 2016, 42, 174-178.

(33) Bampoulis, P.; Sotthewes, K.; Siekman, M. H.; Zandvliet, H. J. W.; Poelsema, B. Graphene Visualizes the Ion Distribution on AirCleaved Mica. Sci. Rep. 2017, 7, 43451.

(34) Giannazzo, F.; Fisichella, G.; Greco, G.; Di Franco, S.; Deretzis, I.; La Magna, A.; Bongiorno, C.; Nicotra, G.; Spinella, C.; Scopelliti, M.; et al. Ambipolar $\mathrm{MoS}_{2}$ Transistors by Nanoscale Tailoring of Schottky Barrier Using Oxygen Plasma Functionalization. ACS Appl. Mater. Interfaces 2017, 9, 23164-23174.

(35) Sotthewes, K.; Bampoulis, P.; Zandvliet, H. J. W.; Lohse, D.; Poelsema, B. Pressure-Induced Melting of Confined Ice. ACS Nano 2017, 11, 12723-12731.

(36) Zhou, W.; Zou, X.; Najmaei, S.; Liu, Z.; Shi, Y.; Kong, J.; Lou, J.; Ajayan, P. M.; Yakobson, B. I.; Idrobo, J.-C. Intrinsic Structural Defects in Monolayer Molybdenum Disulfide. Nano Lett. 2013, 13, 2615.

(37) Kc, S.; Longo, R. C.; Addou, R.; Wallace, R. M.; Cho, K. Impact of Intrinsic Atomic Defects on the Electronic Structure of $\mathrm{MoS}_{2}$ Monolayers. Nanotechnology 2014, 25, 375703.
(38) Guo, Y.; Liu, D.; Robertson, J. Chalcogen vacancies in monolayer transition metal dichalcogenides and Fermi level pinning at contacts. Appl. Phys. Lett. 2015, 106, 173106.

(39) Inoue, A.; Komori, T.; Shudo, K.-i. Atomic-Scale Structures and Electronic States of Defects on $\mathrm{Ar}^{+}$-Ion irradiated $\mathrm{MoS}_{2}$. J. Electron Spectrosc. Relat. Phenom. 2013, 189, 11-18.

(40) Rosenberger, M. R.; Chuang, H.-J.; McCreary, K. M.; Li, C. H.; Jonker, B. T. Electrical Characterization of Discrete Defects and Impact of Defect Density on Photoluminescence in Monolayer WS2. ACS Nano 2018, 12, 1793-1800.

(41) Whangbo, M.-H.; Ren, J.; Magonov, S. N.; Bengel, H.; Parkinson, B. A.; Suna, A. On the Correlation between the Scanning Tunneling Microscopy Image Imperfections and Point Defects of Layered Chalcogenides $2 \mathrm{HMX}_{2}(\mathrm{M}=\mathrm{Mo}, \mathrm{W} ; \mathrm{X}=\mathrm{S}, \mathrm{Se})$. Surf. Sci. 1995, 326, 311-326.

(42) Yankowitz, M.; McKenzie, D.; LeRoy, B. J. Local Spectroscopic Characterization of Spin and Layer Polarization in $\mathrm{WSe}_{2}$. Phys. Rev. Lett. 2015, 115, 136803.

(43) Murata, H.; Kataoka, K.; Koma, A. Scanning Tunneling Microscope Images of Locally Modulated Structures in Layered Materials, $\mathrm{MoS}_{2}(0001)$ and $\mathrm{MoSe}_{2}(0001)$, Induced by Impurity Atoms. Surf. Sci. 2001, 478, 131-144.

(44) Magonov, S. N.; Cantow, H.-J.; Whangbo, M.-H. On the Nature of Nanometer-scale Ring Structures in the Scanning Tunneling Microscopy Imagesof Tungsten Diselenide $\mathrm{WS}_{2}$. Surf. Sci. 1994, 318, L1175-L1180.

(45) Koenraad, P. M.; Flatté, M. E. Single Dopants in Semiconductors. Nat. Mater. 2011, 10, 91.

(46) Sinthiptharakoon, K.; Schofield, S. R.; Studer, P.; Brázdová, V.; Hirjibehedin, C. F.; Bowler, D. R.; Curson, N. J. Investigating Individual Arsenic Dopant Atoms in Silicon Using Low-Temperature Scanning Tunnelling Microscopy. J. Phys. Condens. Matter 2014, 26, 012001.

(47) Pandey, M.; Rasmussen, F. A.; Kuhar, K.; Olsen, T.; Jacobsen, K. W.; Thygesen, K. S. Defect-Tolerant Monolayer Transition Metal Dichalcogenides. Nano Lett. 2016, 16, 2234-2239.

(48) Khan, M. A.; Erementchouk, M.; Hendrickson, J.; Leuenberger, M. N. Electronic and Optical Properties of Vacancy Defects in SingleLayer Transition Metal Dichalcogenides. Phys. Rev. B 2017, 95, 245435.

(49) Salehi, S.; Saffarzadeh, A. Optoelectronic Properties of Defective $\mathrm{MoS}_{2}$ and $\mathrm{WS}_{2}$ Monolayers. J. Phys. Chem. Solids 2018, 121, 172-176.

(50) Komsa, H.-P.; Kotakoski, J.; Kurasch, S.; Lehtinen, O.; Kaiser, U.; Krasheninnikov, A. V. Two-Dimensional Transition Metal Dichalcogenides under Electron Irradiation: Defect Production and Doping. Phys. Rev. Lett. 2012, 109, 035503.

(51) Carvalho, A.; Neto, A. H. C. Donor and Acceptor Levels in Semiconducting Transition-Metal Dichalcogenides. Phys. Rev. B: Condens. Matter Mater. Phys. 2014, 89, 081406.

(52) Haldar, S.; Vovusha, H.; Yadav, M. K.; Eriksson, O.; Sanyal, B. Systematic study of structural, electronic, and optical properties of atomic-scale defects in the two-dimensional transition metal dichalcogenides $\mathrm{MX}_{2}(, M=\mathrm{Mo} \mathrm{W} ;, X=\mathrm{S} \mathrm{Se}, \mathrm{Te})$. Phys. Rev. B: Condens. Matter Mater. Phys. 2015, 92, 235408.

(53) Tung, R. T. The Physics and Chemistry of the Schottky Barrier Height. Appl. Phys. Rev. 2014, 1, 011304.

(54) Roderick, E.; Williams, R. Metal-Semiconductor Contacts; Clarendon Press, Oxford, 1988.

(55) Hertz, H. Uber die Beruhrung fester elastischer Korper. J. für die Reine Angewandte Math. 1881, 92, 156-171.

(56) Barthel, E. In Contact, Interactions, and Dynamics in Acoustic Scanning Probe Microscopy; Marinello, F., Passeri, D., Savio, E., Eds.; Springer-Verlag Berlin, 2013.

(57) Jiao, Y.; Hellman, A.; Fang, Y.; Gao, S.; Käll, M. Schottky Barrier Formation and Band Bending Revealed by First-Principles Calculations. Sci. Rep. 2015, 5, 11374. 
(58) Mönch, W. Valence-Band Offsets and Schottky Barrier Heights of Layered Semiconductors Explained by Interface-Induced Gap States. Appl. Phys. Lett. 1998, 72, 1899-1901.

(59) Kang, J.; Liu, W.; Sarkar, D.; Jena, D.; Banerjee, K. Computational Study of Metal Contacts to Monolayer TransitionMetal Dichalcogenide Semiconductor. Phys. Rev. X 2014, 4, 031005.

(60) Mönch, W. Explanation of the Barrier Heights of Graphene Schottky Contacts by the MIGS-and-Electronegativity Concept. J. Appl. Phys. 2016, 120, 104501.

(61) Mönch, W. Chemical Trends of Barrier Heights in MetalSemiconductor Contacts: On the Theory of the Slope Parameter. Appl. Surf. Sci. 1996, 92, 367-371.

(62) Robertson, J. Band offsets of wide-band-gap oxides and implications for future electronic devices. J. Vac. Sci. Technol., B: Microelectron. Nanometer Struct.-Process., Meas., Phenom. 2000, 18, $1785-1791$.

(63) Rasmussen, F. A.; Thygesen, K. S. Computational 2D Materials Database: Electronic Structure of Transition-Metal Dichalcogenides and Oxides. J. Phys. Chem. C 2015, 119, 13169-13183.

(64) Guo, Y.; Robertson, J. Band engineering in transition metal dichalcogenides: Stacked versus lateral heterostructures. Appl. Phys. Lett. 2016, 108, 233104.

(65) Brudnyi, V. N.; Sarkisov, S. Y.; Kosobutsky, A. V. Electronic properties of $\mathrm{GaSe}$, InSe, GaS and GaTe layered semiconductors: charge neutrality level and interface barrier heights. Semicond. Sci. Technol. 2015, 30, 115019.

(66) Schlüter, M. Chemical Trends in Metal-Semiconductor Barrier Heights. Phys. Rev. B: Condens. Matter Mater. Phys. 1978, 17, 50445047.

(67) Tsugawa, K.; Noda, H.; Hirose, K.; Kawarada, H. Schottky Barrier Heights, Carrier Density, and Negative Electron Affinity of Hydrogen-Terminated Diamond. Phys. Rev. B: Condens. Matter Mater. Phys. 2010, 81, 045303.

(68) Pan, Y.; Li, S.; Ye, M.; Quhe, R.; Song, Z.; Wang, Y.; Zheng, J.; Pan, F.; Guo, W.; Yang, J.; et al. Interfacial Properties of Monolayer $\mathrm{MoSe}_{2}$-Metal Contacts. J. Phys. Chem. C 2016, 120, 13063-13070.

(69) Tersoff, J. Calculation of Schottky Barrier Heights from Semiconductor Band Structures. Surf. Sci. 1986, 168, 275-284.

(70) Mönch, W. Metal-Semiconductor Contacts: Electronic Properties. Surf. Sci. 1994, 299-300, 928-944.

(71) Mönch, W. Role of Virtual Gap States and Defects in MetalSemiconductor Contacts. Phys. Rev. Lett. 1987, 58, 1260-1263.

(72) Bell, M. G.; Liang, W. Y. Electron Energy Loss Studies in Solids; The Transition Metal Dichalcogenides. Adv. Phys. 1976, 25, 53-86.

(73) Laturia, A.; Van de Put, M. L.; Vandenberghe, W. G. Dielectric Properties of Hexagonal Boron Nitride and Transition Metal Dichalcogenides: from Monolayer to Bulk. npj 2D Mater. Appl. 2018, 2, 6 .

(74) Ihm, J.; Louie, S. G.; Cohen, M. L. Diamond-Metal Interfaces and the Theory of Schottky Barriers. Phys. Rev. Lett. 1978, 40, 12081211.

(75) Han, Y.; Wu, Z.; Xu, S.; Chen, X.; Wang, L.; Wang, Y.; Xiong, W.; Han, T.; Ye, W.; Lin, J.; et al. Probing Defect-Induced Midgap States in $\mathrm{MoS}_{2}$ Through Graphene- $\mathrm{MoS}_{2}$ Heterostructures. Adv. Mater. Interfaces 2015, 2, 1500064.

(76) Qiu, H.; Xu, T.; Wang, Z.; Ren, W.; Nan, H.; Ni, Z.; Chen, Q.; Yuan, S.; Miao, F.; Song, F.; et al. Hopping Transport Through Defect-Induced Localized States in Molybdenum Disulphide. Nat. Commun. 2013, 4, 2642.

(77) Feng, L.-P.; Su, J. Effect of Vacancies on Structural, Electronic and Optical Properties of Monolayer $\mathrm{MoS}_{2}$ : A first-Principles Study. J. Alloy. Comp. 2014, 613, 122-127.

(78) Liu, Y.; Stradins, P.; Wei, S.-H. Van der Waals MetalSemiconductor Junction: Weak Fermi Level Pinning Enables Effective Tuning of Schottky Barrier. Sci. Adv. 2016, 2, No. e1600069.

(79) Chanana, A.; Mahapatra, S. Prospects of Zero Schottky Barrier Height in a Graphene-Inserted $\mathrm{MoS}_{2}$-Metal Interface. J. Appl. Phys. 2016, 119, 014303. 